\title{
Clinical associations between gout and multiple sclerosis, Parkinson's disease and motor neuron disease: record-linkage studies
}

\author{
Julia Pakpoor ${ }^{1}$, Olena O Seminog ${ }^{1}$, Sreeram V Ramagopalan² and Michael J Goldacre ${ }^{1^{*}}$
}

\begin{abstract}
Background: Uric acid has antioxidant effects on neurons. Abnormally high levels of uric acid are, however, associated with gout. Previous studies have suggested that high levels of uric acid (and the presence of gout) may exert a protective effect against the risk of developing some neurological diseases. We aimed to investigate this hypothesis in a large database of hospital admissions in England.

Methods: We analysed a database of linked statistical records of hospital admissions and death registrations in England (1999-2012). A cohort of people with gout was constructed and followed for development of multiple sclerosis (MS), Parkinson's disease (PD) or motor neuron disease (MND). Then, conversely, cohorts of all people in the database with MS, PD or MND were constructed and followed for subsequent gout. Rate ratios (RRs) were determined, comparing these cohorts with people in a reference cohort.

Results: In the gout cohort, we observed a modest elevation of the overall risk of subsequent MS, PD and MND (respectively, RR $=1.27$ (95\% confidence interval 1.03-1.55), 1.11 (1.05-1.17) and 1.28 (1.11-1.48) which was largely attributable to an increased risk observed in the early years after hospitalisation for gout. The increased risk of neurological disease did not remain after 5 years. In the cohorts of people with MS or PD, there was a significantly reduced risk of subsequent gout admission ( $R R=0.79$ (0.69-0.89) and 0.83 (0.79-0.87), respectively). This inverse association was sustained over time. There was also a reduced risk of MND following gout which only emerged more than five years following initial gout admission (RR at $5+$ years $0.35(0.15-0.68)$ ).

Conclusions: This study investigated the epidemiological evidence for a protective role of high serum concentration of uric acid, for which we used gout as a proxy, in the aetiology of MS, PD or MND. Our observations do not support this hypothesis. However, when the order was reversed, and we retrospectively followed up patients with MS, PD and MND for a number of years, we found a statistically significant deficit of gout. This suggests that there is relationship between some aspects of these neurodegenerative diseases and metabolism of uric acid.
\end{abstract}

Keywords: Multiple sclerosis, Parkinson's disease, Motor neuron disease, Uric acid, Gout

\section{Background}

Oxidative stress is thought to have an important role in neuronal degeneration. Neuronal degeneration is a characteristic feature of several neurological disorders including multiple sclerosis (MS), Parkinson's disease (PD), and motor neuron disease (MND) [1,2]. A high fatty acid content and a low antioxidant capacity are

\footnotetext{
* Correspondence: michael.goldacre@dph.ox.ac.uk

'Unit of Health-Care Epidemiology, Nuffield Department of Population Health, University of Oxford, Oxford OX3 7LF, UK

Full list of author information is available at the end of the article
}

thought to be factors which render neural cells more susceptible than other body tissues to oxidative stress. This, in turn, may result in damage to proteins and DNA, subsequent inflammation and neuronal loss [3-5]. Uric acid is a potent antioxidant found throughout extracellular fluid, as sodium urate, and is thought to account for more than half of the antioxidant capacity of plasma [6]. Levels of serum urate are closely correlated with levels of urate in the cerebrospinal fluid (CSF) and CSF urate is typically $10 \%$ of peripheral levels. This suggests that CSF levels of urate are in part dependent on 
serum uric acid levels [7]. It has been suggested that uric acid may have neuroprotective influence as a scavenger of reactive nitrogen and oxygen radicals. A powerful antioxidant effect of uric acid on neurons has been demonstrated through in vivo and in vitro studies $[8,9]$. The hypothesis that high levels of uric acid exert a protective effect against oxidative neuronal damage is supported by studies showing that individuals with PD, MS and MND have significantly lower serum uric acid levels than healthy controls [10-12]; and there is some evidence that low uric acid levels correlate with progression of symptoms and disease relapse $[13,14]$. Among men participating in a $30-$ year prospective study, men with elevated uric acid levels had a $40 \%$ reduction in the incidence of PD, unaffected by adjustment for age and smoking [15]. Similarly, a large nested case-control study found that higher plasma concentrations of urate predict risk of PD, with a significantly reduced rate of PD for men in the highest quartile of uricemia compared with the lowest [16]. This notion is strengthened by the finding that a diet which increases plasma urate levels is associated with a reduced PD risk, rendering it unlikely that the association is merely a consequence of shared predisposing factors [17].

Abnormally high levels of serum uric acid are, however, known to be pathological and most commonly associated with gout, a form of recurrent acute inflammatory arthritis in which uric acid crystallizes and is deposited within joints [18]. Individuals with gout, and therefore high levels of serum uric acid, have been postulated to have greater protection from neurodegenerative diseases in which oxidative stress is thought to contribute to pathogenesis. A lower risk of PD and MS in individuals with gout has been previously reported [19-21]. The relationship between gout (as an indicator of high levels of uric acid) and the incidence of neurodegenerative diseases is of potential therapeutic interest given the proposed possibility that elevation of uric acid levels may reduce the incidence of neurological disease. However, it is currently unclear if lower levels of uric acid in individuals with MS, PD and MND represent a cause or consequence of the disease. This record linkage study therefore aimed to extend previous findings by determining the risk of PD, MS and MND in individuals with a history of gout, and, conversely, the risk of subsequent gout in individuals with PD, MS or MND.

\section{Methods}

\section{Population and data}

We used a linked English national data set of hospital admissions (Hospital Episode Statistics (HES) and mortality. HES data are records of hospital care that are compiled for every episode of hospital day case care (when the patient is admitted to hospital but does not stay overnight) or hospital inpatient care in all English National Health Service (NHS) hospitals, and they were supplied by the English national Information Centre for Health and Social Care. The mortality data, derived from death registrations, were supplied by the Office for National Statistics. The linked dataset used in this study, in which successive records for each individual were linked together, was constructed by the Oxford record linkage group. The International Classification of Disease (ICD) codes used for gout were 274 in the ninth revision and M10 in the tenth. The ICD 9 and 10 revision codes used for MS, PD, and MND were, respectively, 340 and G35, 332.0 and G20, and 335.2 and G12.2.

\section{Gout before MS, PD, or MND}

The methods of analysis were the same for all neurological diseases; we describe the methods for gout followed by MS as the example. A cohort of people with gout (the 'exposure' cohort) was constructed for people with a diagnosis of gout in an episode of hospital care by identifying the first episode of day case care, or admission, for gout during the study period. A reference cohort was constructed by identifying the first admission for each individual with various other mainly minor medical and surgical conditions as described in previous studies of disease associations [22]. Standard epidemiological practice was followed by selecting a diverse range of conditions rather than relying on a limited range (in case the latter are themselves atypical in their risk of neurological disease). As a check, we estimated the risk of each neurological disease in the reference cohort to ensure that it does not include conditions that have atypically high or low rates of neurological disease.

People were included in the gout or reference cohort if they did not have an admission for MS either before or at the same time as the admission for gout or the reference condition. The database was then investigated for any subsequent NHS hospital care for, or death from, MS in these cohorts (the 'outcome' condition). We considered that rates of MS in the reference cohort would approximate those in the general population while allowing for migration (data on migration by individuals covered by the datasets was not available).

\section{Statistical methods}

Rates of MS were calculated based on person-days. Date of entry into each cohort was the date of first admission for gout, or reference condition, from the start of data collection on $1^{\text {st }}$ January1999 and date of exit was the date of first record of MS, death, or the end of data collection (31 ${ }^{\text {st }}$ March 2012), whichever was the earliest. We calculated rates for MS, stratified and then standardised by age (in five-year age groups), sex, calendar year of first recorded admission, region of residence, and quintile of patients' Index of Deprivation score (a standard English measure of socio-economic status). The indirect method 
of standardisation was used, with the combined gout and reference cohorts as the standard population. We applied the stratum-specific rates (e.g. within each five-year age stratum) in the standard population to the number of people in each stratum (e.g. within the same five-year age stratum) in the gout cohort and then, separately, to those in the same stratum in the reference cohort, to obtain the expected number of people with MS in each stratum of the gout and reference cohort. Observed and expected numbers were then summed across all strata to give totals for all strata combined. Rate ratios (RRs) were calculated by taking the standardised rate of occurrence of MS in the gout cohort relative to the reference cohort using the formula $\left(\mathrm{O}^{\text {gout }} / \mathrm{E}^{\text {gout }}\right) /\left(\mathrm{O}^{\text {ref }} / \mathrm{E}^{\text {ref }}\right)$, where $\mathrm{O}$ and $\mathrm{E}$ are the observed and expected numbers of MS cases in the gout and reference cohorts, respectively. The 95\% confidence interval $(95 \% \mathrm{CI})$ for the rate ratio of MS and $\chi^{2}$ statistics for its significance were calculated, with continuity correction for small numbers, as described elsewhere [23].

\section{MS, PD, and MND before gout}

We then used the same methods to study the neurological diseases followed by gout. We constructed 'exposure' cohorts of people, separately, comprising all people in the dataset with MS, PD, or MND. We compared them with the reference cohort. In this analysis, we excluded anyone with gout prior to or at the same time as their first admission for neurological disease or reference cohort condition. Taking both study designs - gout followed by neurological disease, and neurological disease followed by gout - the exclusions ensured that no individual was double-counted as a case of gout both before and after neurological disease.

The datasets are in the custodianship of the Unit of Health-Care Epidemiology and are not freely available. Ethical approval to construct, maintain, develop and analyse the datasets has been obtained, on an ongoing basis, from the Central and South Bristol Multi-Centre Research Ethics Committee (04/Q2006/176).

\section{Results}

Gout before MS, PD or MND

Basic demographic information of the disease cohorts is shown in Table 1. The number of people admitted to hospital with gout was 214,653. There were approximately 9 million people in the reference cohort. Comparing the gout cohort with the reference cohort, the rate ratios (RR) for subsequent MS, PD and MND were, respectively, 1.27 (95\% confidence interval 1.03-1.55), 1.11 (1.05-1.17) and 1.28 (1.11-1.48), Table 2. Results were no longer significant upon inclusion only of cases of neurological disease which occurred at least five years following initial gout admission (Table 2).

There were $82,220,217,179$ and 25,185 people, respectively, in the MS, PD and MND cohorts. In the cohorts of people with MS, PD and MND, the RRs for subsequent gout were, respectively, 0.79 (0.69-0.89), 0.83 (0.79-0.87) and 0.94 (0.75-1.16), Table 3. Notably, a reduced risk of MS and PD following gout was significant after the first year following admission for gout, and was then sustained long-term. For example, after 5 years following hospitalisation for MS the rate ratio for gout was $0.70(0.57-0.85)$ and after hospitalisation for PD 0.52 (0.45-0.59). The risk for gout was not different between the reference cohort and people hospitalised with MND within five years after hospitalisation for MND, but it was significantly and strikingly low at 5 years and more after hospitalisation (RR 0.35, 0.15-0.68), Table 3.

\section{Discussion}

In the gout cohort, we demonstrated a statistically significant but modest elevation of risk of admission for subsequent MS, PD and MND. After excluding cases of MS, PD or MND observed in the first year after hospitalisation with gout, there was no elevated RR for these conditions. These results do not confirm findings from previous studies suggesting a reduced risk of neurological disease following gout as a consequence of a neuroprotective antioxidant effect of uric acid, or the corollary that individuals with low levels of uric acid have an impaired

Table 1 Demographic characteristics of disease cohorts

\begin{tabular}{|c|c|c|c|c|c|c|c|c|}
\hline \multirow{2}{*}{$\begin{array}{l}\text { Age } \\
\text { (years) }\end{array}$} & \multicolumn{2}{|l|}{ Gout } & \multicolumn{2}{|c|}{ Multiple sclerosis } & \multicolumn{2}{|c|}{ Parkinson's disease } & \multicolumn{2}{|c|}{ Motor neuron disease } \\
\hline & Age (\%) & Male (\%) & Age (\%) & Male (\%) & Age (\%) & Male (\%) & Age (\%) & Male (\%) \\
\hline$<25$ & 0.1 & 76.7 & 2.1 & 30.4 & 0 & 53.7 & 1.6 & 64.0 \\
\hline $25-44$ & 4.9 & 92.4 & 29.1 & 29.3 & 0.5 & 57.8 & 4.1 & 64.9 \\
\hline $45-54$ & 9.0 & 91.1 & 25.6 & 29.7 & 1.5 & 61.9 & 8.0 & 63.0 \\
\hline $55-64$ & 15.7 & 86.9 & 21.9 & 33.7 & 6.2 & 63.7 & 19.3 & 59.9 \\
\hline 65-74 & 23.9 & 78.5 & 13.3 & 32.6 & 22.8 & 61.3 & 31.6 & 56.8 \\
\hline $75+$ & 46.3 & 61.4 & 8.1 & 28.2 & 68.9 & 53.6 & 35.4 & 53.0 \\
\hline Total & 100 & 73.7 & 100 & 30.8 & 100 & 56.2 & 100 & 57.0 \\
\hline
\end{tabular}


Table 2 Occurrence of multiple sclerosis (MS), Parkinson's disease (PD) and motor neuron disease (MND) in patients hospitalised with gout by time interval: observed $(0)$ and expected (E) number of cases in each cohort, relative risk (RR) with $95 \%$ confidence intervals $(95 \% \mathrm{CI})$ in the exposure cohort compared with the reference cohort ${ }^{\mathrm{a}}$

\begin{tabular}{|c|c|c|c|c|c|c|c|c|c|}
\hline \multirow{2}{*}{$\begin{array}{l}\text { Time } \\
\text { interval }\end{array}$} & \multicolumn{3}{|c|}{ Gout before MS } & \multicolumn{3}{|c|}{ Gout before PD } & \multicolumn{3}{|c|}{ Gout before MND } \\
\hline & 0 & $E$ & RR $(95 \% \mathrm{Cl})$ & 0 & $E$ & $\mathrm{RR}(95 \% \mathrm{Cl})$ & 0 & $E$ & $\mathrm{RR}(95 \% \mathrm{Cl})$ \\
\hline Overall & 98 & 77.3 & $1.27(1.03-1.55)$ & 1568 & 1420.3 & $1.11(1.05-1.17)$ & 197 & 155.1 & $1.28(1.11-1.48)$ \\
\hline$<1 \mathrm{yr}$ & 36 & 23.5 & $1.55(1.08-2.15)$ & 405 & 330.9 & $1.24(1.12-1.38)$ & 59 & 39.0 & $1.56(1.17-2.04)$ \\
\hline $1-4$ yrs & 39 & 36.9 & $1.06(0.75-1.45)$ & 824 & 745.8 & $1.11(1.03-1.19)$ & 95 & 80.4 & $1.19(0.96-1.46)$ \\
\hline $5+y r s$ & 23 & 16.8 & $1.37(0.87-2.06)$ & 339 & 343.6 & $0.99(0.88-1.10)$ & 43 & 35.6 & $1.21(0.87-1.64)$ \\
\hline
\end{tabular}

${ }^{a}$ Conditions used in reference cohort, with Office of Population, Censuses and Surveys (OPCS) code edition 4 for operations and ICD10 code for diagnosis (with equivalent codes used for other coding editions): adenoidectomy (OPCS4 E20), appendectomy (H01-H03), dilation and curettage (Q10-Q11), hip replacement (W37-W39), knee replacement (W40- W42), cataract (H25), otitis (H60-H67), upper respiratory tract infections (J00-J06), varicose veins (I83), haemorrhoids (I84), deflected septum (J34.2), nasal polyp (J33), impacted tooth and other disorders of teeth (K00-K03), inguinal hernia (K40), in-growing nail, toenail and other diseases of nails (L60), bunion (M20.1), internal derangement of knee (M23), selected limb fractures (S42, S52, S62, S82, S92), contraceptive management (Z30).

Note that, in analysis, we included all people eligible to be in the reference cohort in each stratum and calculated the observed and expected number of people within each age stratum (see Method). Then, given that the individual stratum-specific values of observed and expected cases were equivalent in respect of age, we summed the age-specific values to provide an age-standardised all-ages set of RRs.

ability to counteract free radical toxicity $[19,20]$. It is possible that the inflammatory state associated with the development of symptomatic gout or recurrent inflammatory arthritis (rather than simply hyperuricemia in previous work) counteracts any potential protective antioxidant effect of uric acid. The observed slightly increased risk in early years might be a reflection of surveillance bias, whereby an individual seen in hospital for one condition is more likely to have a second detected. Another possible explanation for the small increase in risk in the early years, that was not sustained long-term, is that individuals admitted to hospital with gout may undertake measures successfully to lower their levels of uric acid (and reduce future gout attacks), which may involve both dietary changes and the use of uric acid reducing medication [24]. Further investigation of the comparison of risk of MS, PD or MND in treated vs untreated hyperuricemia would have enabled better characterisation of the association, but such data was not available in this study.

Further, from hospital admission statistical records alone, we cannot be sure that, in the gout cohort, the onset of gout really did invariably precede the onset of MS, PD and MND. The date of first admission for a neurodegenerative disease is likely to have been preceded by a period of sub-clinical/undiagnosed disease, and thus, the disease state itself may have resulted in somewhat elevated levels of uric acid and an increased risk of gout. The fact that the raised risk was not sustained over time means that we cannot be confident that the findings support a causal role for uric acid in altering neurological disease risk. It needs further investigation to establish whether uric acid levels alter neurological disease risk (and/or disease progression), given the potential therapeutic approach of altering uric acid levels (through either dietary or pharmacological measures) in altering risk of neurological diseases.

In the MS, PD and MND cohorts, we found a prolonged reduced risk of subsequent admission for gout. Notably, for the MS and PD cohorts a reduced risk of gout was seen following the first year of initial admission for neurological disease. In the MND cohort, this reduced risk only appeared five years following initial MND admission. Although, we can only speculate about pathophysiological mechanisms, our findings on gout before and after the neurological diseases might indicate that the causal pathway is that the neurological disease states result in lowered uric acid levels. For example, central nervous system

Table 3 Occurrence of gout in patients hospitalised with multiple sclerosis (MS), Parkinson's disease (PD) and motor neuron disease (MND) by time interval: observed (O) and expected (E) number of cases in each cohort, relative risk (RR) with $95 \%$ confidence intervals $(95 \% \mathrm{CI})$ in the exposure cohort compared with the reference cohort

\begin{tabular}{|c|c|c|c|c|c|c|c|c|c|}
\hline \multirow[b]{2}{*}{ Time interval } & \multicolumn{3}{|c|}{ MS before gout } & \multicolumn{3}{|c|}{ PD before gout } & \multicolumn{3}{|c|}{ MND before gout } \\
\hline & 0 & $\mathrm{E}$ & RR $(95 \% \mathrm{Cl})$ & 0 & $\mathrm{E}$ & RR $(95 \% \mathrm{Cl})$ & 0 & $\mathrm{E}$ & RR $(95 \% \mathrm{Cl})$ \\
\hline Overall & 246 & 311.7 & $0.79(0.69-0.89)$ & 1521 & 1825 & $0.83(0.79-0.87)$ & 83 & 88.4 & $0.94(0.75-1.16)$ \\
\hline$<1 \mathrm{yr}$ & 50 & 43.1 & $1.16(0.86-1.53)$ & 522 & 489 & $1.07(0.98-1.17)$ & 41 & 29.9 & $1.38(0.99-1.87)$ \\
\hline $1-4$ yrs & 96 & 126.4 & $0.76(0.61-0.93)$ & 728 & 893.2 & $0.85(0.79-0.92)$ & 34 & 35.5 & $0.96(0.66-1.34)$ \\
\hline $5+y r s$ & 100 & 142.2 & $0.70(0.57-0.85)$ & 231 & 442.8 & $0.52(0.45-0.59)$ & 8 & 23.0 & $0.35(0.15-0.68)$ \\
\hline
\end{tabular}

See footnotes Table 2 . 
inflammation which is a typical feature of MS has been associated with increased production of the strong oxidant peroxynitrite and free radicals, which may in turn lead to reduced levels of uric acid, which is a peroxynitrite scavenger [25]. A cross-sectional study which found markedly reduced levels of serum urate in MND patients with bulbar onset, and with a longer duration of disease, suggested that the reduced levels of serum urate may be a consequence of malnutrition secondary to the impact of MND, particularly in its later stages [26].

This study's strengths include its large size and the fact that it is a national study and therefore generalizable as the cohorts are likely to relatively accurately reflect the national prevalence of these neurological disorders. Further, it demonstrates what can be done with the analysis of readily available electronic medical records.

The study also has limitations. Importantly, it is not a cohort study with follow-up from the date of "first ever" diagnosis, but just from "first recorded" diagnosis in a hospital day case or inpatient record. Data are not available on patients who migrate out of England. The dataset is limited to people who were admitted to hospital, or who received day case specialist care, and thus there exists the potential for selection bias in omitting milder cases of gout. Further, given that only a minority of individuals with elevated uric acid levels develop gout, using gout as a proxy for high uric acid levels may underestimate the association between uric acid and neurological diseases. Uric acid levels appear positively correlated with risk of gout, and the gout cohort is therefore more likely to capture those with more severe uric acid elevation in which, over time, urate crystals have deposited in or around joints. There is very limited information on potential confounding factors such as detailed socioeconomic characteristics, beyond the IMD index, ethnicity and smoking.

Studies of alternative design would be costly and complex undertakings. For example, if funds allowed, "shoeleather" studies of individual patients could be undertaken by personal follow-up, such as following large numbers of people with gout to await development of these three uncommon neurological diseases, or studies could be undertaken of people with MS, PD or MND to identify clinical gout or to measure their serum uric acid levels. The latter would need to be designed in ways that allow the investigators to determine the time sequence of 'high levels of uric acid before the neurological condition' or 'neurological condition before changes in uric acid levels'.

\section{Conclusions}

In summary, the data in our study provide good evidence of an inverse association between gout and MS $\mathrm{PD}$, and MND. Our data therefore supports the notion that the pre-existence of the neurological diseases reduces the occurrence of gout. We highlight the need for future studies to incorporate duration of disease, and effects of treatment, with longitudinal data on uric acid levels to determine whether altered uric acid levels, associated with neurodegenerative disease, are truly a cause or a consequence of neurodegenerative disease.

\section{Competing interests}

The authors declare that they have no competing interests.

\section{Authors' contributions}

OOS proposed the study and undertook the analysis. MJG designed the analysis. JP and SVR wrote the first draft. All authors contributed to the interpretation of the data and to the final draft.

\section{Acknowledgements}

The Unit of Health-Care Epidemiology and its work on record linkage were funded by the English National Institute for Health Research. The Wellcome Trust provided core support to SVR (Grant 075491/Z/04) when he was working at the Wellcome Trust Centre for Human Genetics. MJG is part-funded by Public Health England. The study sponsors had no role in the design and conduct of the study; collection, management, analysis, and interpretation of the data; and preparation, review, or approval of the manuscript. All authors state that this research was carried out independently of the influence of funding bodies.

\section{Author details}

${ }^{1}$ Unit of Health-Care Epidemiology, Nuffield Department of Population Health, University of Oxford, Oxford OX3 7LF, UK. '²epartment of Physiology, Anatomy and Genetics and Medical Research Council Functional Genomics Unit, University of Oxford, Oxford, UK.

Received: 6 November 2013 Accepted: 18 February 2015

Published online: 28 February 2015

References

1. Jenner P. Oxidative stress in Parkinson's disease. Ann Neurol. 2003;53 suppl 3:526-38.

2. Gilgun-Sherki Y, Melamed E, Offen D. The role of oxidative stress in the pathogenesis of multiple sclerosis: the need for effective antioxidant therapy. J Neurol. 2004;251:261-8

3. Alam ZI, Jenner A, Daniel SE, Lees AJ, Cairns N, Marsden CD, et al. Oxidative DNA damage in the parkinsonian brain: an apparent selective increase in 8-hydroxyguanine levels in substantia nigra. J Neurochem. 1997;69:1196-203.

4. Dexter DT, Holley AE, Flitter WD, Slater TF, Wells FR, Daniel SE, et al. Increased levels of lipid hydroperoxides in the parkinsonian substantia nigra: an HPLC and ESR study. Mov Disord. 1994;9:92-7.

5. Floyd RA, Carney JM. Free radical damage to protein and DNA: mechanisms involved and relevant observations on brain undergoing oxidative stress. Ann Neurol. 1992;32(Suppl):S22-7.

6. Yeum KJ, Russell RM, Krinsky NI, Aldini G. Biomarkers of antioxidant capacity in the hydrophilic and lipophilic compartments of human plasma. Arch Biochem Biophys. 2004;430(1):97-103.

7. Becker BF, Kastenbauer S, Kodel U, KiesI D, Pfister HW. Urate oxidation in CSF and blood of patients with inflammatory disorders of the nervous system. Nucleosides Nucleotides Nucleic Acids. 2004;23(8-9):1201-4.

8. Duan W, Ladenheim B, Cutler RG, Kruman II, Cadet JL, Mattson MP. Dietary folate deficiency and elevated homocysteine levels endanger dopaminergic neurons in models of Parkinson's disease. J Neurochem. 2002;80:101-10.

9. Church WH, Ward VL. Uric acid is reduced in the substantia nigra in Parkinson's disease: effect on dopamine oxidation. Brain Res Bull. 1994;33:419-25.

10. Rentzos M, Nikolaou C, Anagnostouli M, Rombos A, Tsakanikas K, Economou $M$, et al. Serum uric acid and multiple sclerosis. Clin Neurol Neurosurg. 2006:108:527-31.

11. de Lau LML, Koudstaal PJ, Hofman A, Breteler MM. Serum uric acid levels and the risk of Parkinson disease. Ann Neurol. 2005:58:797-800.

12. Keizman D, Ish-Shalom M, Berliner S, Maimon N, Vered $Y$, Artamonov I, et al. Low uric acid levels in serum of patients with ALS: further evidence for oxidative stress? J Neurol Sci. 2009;285(1-2):95-9. 
13. Toncev G, Milicic B, Toncev S, Samardzic G. Serum uric acid levels in multiple sclerosis patients correlate with activity of disease and blood-brain barrier dysfunction. Eur J Neurol. 2002;9:221-6.

14. Ascherio A, LeWitt PA, Xu K, Eberly S, Watts A, Matson WR, et al. DATATOP Investigators: Urate as a predictor of the rate of clinical decline in Parkinson disease. Arch Neurol. 2009;66(12):1460-8.

15. Davis JW, Grandinetti A, Waslien Cl, Ross GW, White LR, Morens DM. Observations on serum uric acid levels and the risk of idiopathic Parkinson's disease. Am J Epidemiol. 1996;144(5):480-4.

16. Weisskopf MG, O'Reilly E, Chen H, Schwarzschild MA, Ascherio A. Plasma urate and risk of Parkinson's disease. Am J Epidemiol. 2007;166(5):561-7.

17. Gao X, Chen H, Choi HK, Curhan G, Schwarzschild MA, Ascherio A. Diet, urate, and Parkinson's disease risk in men. Am J Epidemiol. 2008;167(7):831-8.

18. Choi HK, Curhan G. Gout: epidemiology and lifestyle choices. Curr Opin Rheumatol. 2005;17:341-5.

19. Alonso A, Rodríguez LA, Logroscino G. Gout and risk of Parkinson disease: a prospective study. Neurology. 2007;69(17):1696-700.

20. Hooper DC, Spitsin S, Kean RB, Champion JM, Dickson GM, Chaudhry I, et al Uric acid, a natural scavenger of peroxynitrite, in experimental allergic encephalomyelitis and multiple sclerosis. Proc Natl Acad Sci U S A. 1998;95(2):675-80

21. De Vera M, Rahman MM, Rankin J, Kopec J, Gao X, Choi H. Gout and the risk of Parkinson's disease: a cohort study. Arthritis Rheum. 2008;59(11):1549-54.

22. Seminog OO, Goldacre MJ. Risk of benign tumours of nervous system, and of malignant neoplasms, in people with neurofibromatosis: population-based record-linkage study. Br J Cancer. 2013;108:193-8.

23. Breslow NE, Day NE, Day NE. Statistical methods in cancer research. Volume II-The design and analysis of cohort studies. IARC Sci. 1987;82:1-406.

24. Emmerson BT. The management of gout. N Engl J Med. 1996;334:445-51.

25. Pacher P, Beckman JS, Liaudet L. Nitric oxide and peroxynitrite in health and disease. Physiol Rev. 2007;87(1):315-424.

26. Zoccolella S, Simone IL, Capozzo R, Tortelli R, Leo A, D’Errico E, et al. An exploratory study of serum urate levels in patients with amyotrophic lateral sclerosis. J Neurol. 2011;258(2):238-43.

\section{Submit your next manuscript to BioMed Central and take full advantage of:}

- Convenient online submission

- Thorough peer review

- No space constraints or color figure charges

- Immediate publication on acceptance

- Inclusion in PubMed, CAS, Scopus and Google Scholar

- Research which is freely available for redistribution 